\title{
Frühe rheumatoide Arthritis - Herausforderungen der Diagnose
}

Bei Patienten mit undifferenzierter Arthritis ist es bekanntlich wichtiger, wann als womit behandelt wird. Denn es gilt, das „,window of opportunity“ zu nutzen. Das hat jedoch Tücken, wie Prof. Dr. Paul Emery, Institute of Rheumatic and MSK Medicine, Leeds University, Großbritannien auf dem diesjährigen Kongress der European League Against Rheumatism (EULAR) in London, berichtete.

Quelle:

Dr. Wiebke Kathmann, SpringerMedizin.de
Die rheumatoide Arthritis (RA) sollte zwar möglichst früh therapiert werden, ist aber so manches Mal im Frühstadium nicht eindeutig zu diagnostizieren. Denn je früher sich der Patient vorstelle, umso schwieriger sei es, die Zahl der betroffenen Gelenke korrekt zu ermitteln und damit die Diagnose zu stellen, so Emery in der HOT-Sitzung zur frühen rheumatoiden

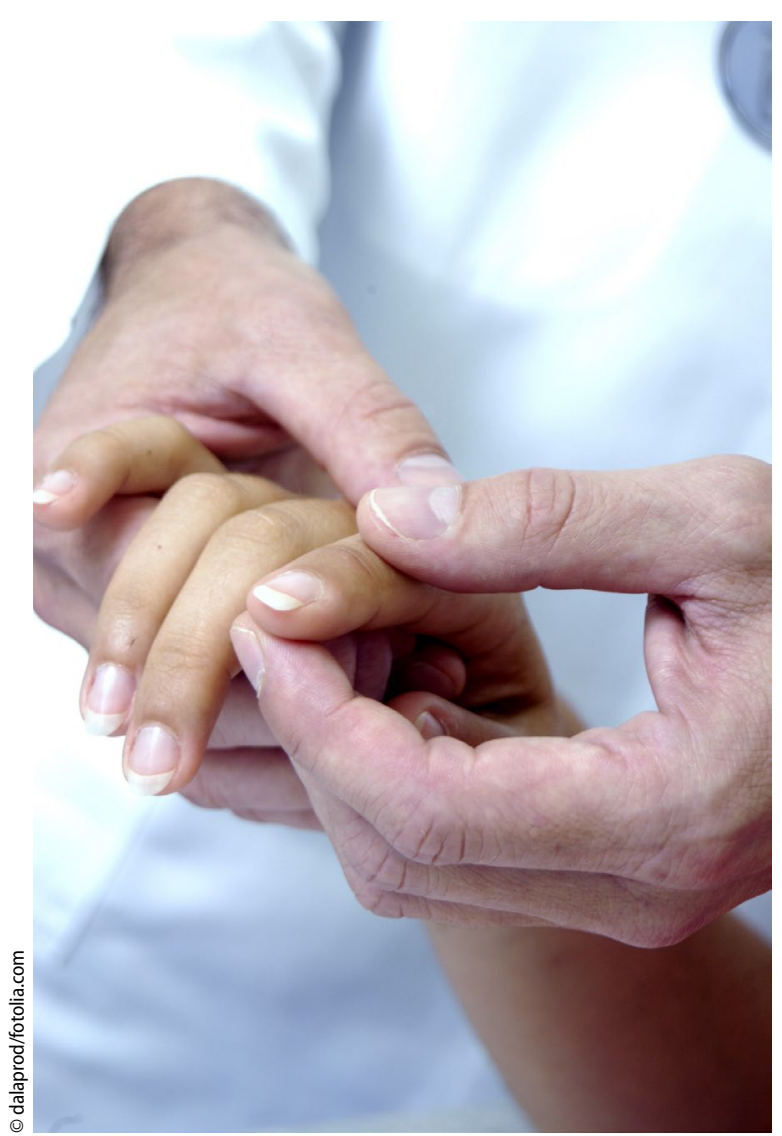

Abb. 1 A Je früher sich der Patient vorstellt, umso schwieriger ist es, die Zahl der betroffenen Gelenke korrekt zu ermitteln
Arthritis. Damit komme dem Nachweis einer Synovitis bei Patienten mit entzündlichen Symptomen und positiver Serologie (Rheumafaktor oder ACPApositiv) eine wichtige Rolle zu, was wiederum eine andere Bildgebung als das Röntgen erforderlich mache.

\section{Aktive Synovitis diagnoseführend} Denn Erosionen sind nur dann prognostisch relevant, wenn sie aktiv sind, also mit einer Synovitis oder einem Knochenödem einhergehen. "Ohne Synovitis keine Erosion und keine Progression", so Emery weiter und damit auch keine Behandlungsnotwendigkeit. Die Synovitis lasse sich am einfachsten mittels Ultraschall ermitteln, das Knochenmarködem mittels Magnetresonanztomographie (MRT). Letzteres könne Erosionen besser und früher detektieren als das Röntgen und erlaube zudem eine positive bzw. negative Vorhersage zum Verlauf.„Finden sich in der MRT zu Beginn keine Erosionen, so haben $82 \%$ der Patienten zwei Jahre später im Röntgen weiterhin keine Erosionen", so Emery.

\) Erosionen sind nur dann prognostisch relevant, wenn sie aktiv sind, also mit einer Synovitis oder einem Knochenödem einhergehen

Ist eine aktive Synovitis nachgewiesen, stellt sich im nächsten Schritt die Frage, ob die Patienten die ACR/EULAR Klassifikationskriterien von 2010 erfüllen. Haben sie im Score weniger als sechs Punkte, kann die Bildgebung dazu genutzt werden, die Zahl der betroffenen Gelenke zu ermitteln. Beträgt der Score dann $\geq 6$, ist gemäß $A C R / E U L A R-K r i t e r i e n$ von einer RA auszugehen. Findet sich in der Ultraschall-Untersuchung keine Synovitis, sollte die Diagnose in Frage gestellt werden.

\) Patienten, die im ersten Jahr mit TNF a-Inhibitoren plus MTX therapiert werden, erreichen in etwa $70 \%$ eine Remission

\section{Immunologisches ,window of opportunity" nutzen}

Zu Beginn des Krankheitsprozesses einer RA ist die Immunologie laut Emery noch relativ normal, was das gute Ansprechen auf MTX bei der frühen RA erkläre. Gelinge es, die Krankheitsaktivität in dieser Phase mittels MTX zu kontrollieren, könne wahrscheinlich auf Biologika verzichtet werden. Darauf folgt offenbar eine TNF a-dominierte Phase. Denn Patienten, die im ersten Jahr mit TNF a-Inhibitoren plus MTX therapiert werden, erreichen in etwa $70 \%$ eine $\mathrm{Re}$ mission. Die frühe TNF $a-G a b e$ sorgt somit langfristig für ein besseres Outcome, wie auch in der MRT gezeigt werden kann: Das Volumen der Synovia verringert sich.

rheuma plus $2016 \cdot 15: 52$ DOI 10.1007/s12688-016-0086-y Online publiziert: 29. August 2016 (c) Springer-Verlag Wien 2016 